\title{
Up-Regulation of hsa-miR-210 Promotes Venous Metastasis and Predicts Poor Prognosis in Hepatocellular Carcinoma
}

\author{
Jia Ji ${ }^{1,2+}$, Yuan Rong ${ }^{1 \dagger}$, Chang-Liang Luo ${ }^{1}$, Shuo $\mathrm{Li}^{1}$, Xiang Jiang ${ }^{3}$, Hong Weng ${ }^{4}$, \\ Hao Chen ${ }^{5}$, Wu-Wen Zhang ${ }^{1}$, Wen $\mathrm{Xie}^{1 *}$ and Fu-Bing Wang ${ }^{1 *}$ \\ ${ }^{1}$ Department of Laboratory Medicine, Zhongnan Hospital of Wuhan University, Wuhan, China, ${ }^{2}$ Department of Laboratory \\ Medicine, Wuhan Children's Hospital, Huazhong University of Science and Technology, Wuhan, China, ${ }^{3}$ Department of \\ Hepatobiliary and Pancreatic Surgery, Zhongnan Hospital of Wuhan University, Wuhan, China, ${ }^{4}$ Center for Evidence-Based \\ and Translational Medicine, Zhongnan Hospital of Wuhan University, Wuhan, China, ${ }^{5}$ Department of Pathology, Zhongnan \\ Hospital of Wuhan University, Wuhan, China
}

OPEN ACCESS

Edited by:

Tao Liu,

University of New South Wales,

Australia

Reviewed by:

Francesca Lovat,

The Ohio State University,

United States

Deborah Stroka,

University of Bern, Switzerland

*Correspondence:

Fu-Bing Wang

wfb20042002@sina.com

Wen Xie

whxw007@126.com

†These authors have contributed equally to this work

Specialty section: This article was submitted to Molecular and Cellular Oncology,

a section of the journal

Frontiers in Oncology

Received: 16 July 2018 Accepted: 13 November 2018 Published: 03 December 2018

Citation:

Ji J, Rong Y, Luo C-L, Li S, Jiang X, Weng $H$, Chen $H$, Zhang $W$-W, Xie $W$ and Wang F-B (2018) Up-Regulation

of hsa-miR-210 Promotes Venous

Metastasis and Predicts Poor

Prognosis in Hepatocellular Carcinoma. Front. Oncol. 8:569. doi: 10.3389/fonc.2018.00569
Objective: To investigate the potential biomarkers for venous metastasis of hepatocellular carcinoma (HCC), and briefly discuss their target genes and the signaling pathways they are involved in.

Materials and Method: The dataset GSE6857 was downloaded from GEO. Significantly differentially expressed miRNAs were identified using the R package "limma," After that, the survival analysis was conducted to discover the significance of these up-regulated miRNAs for the prognosis of HCC patients. Additionally, miRNAs which were up-regulated in venous metastasis positive HCC tissues and were significant for the prognosis of HCC patients were further verified in clinical samples using RT-qPCR. The miRNAs were then analyzed for their correlations with clinical characteristics including survival time, AFP level, pathological grade, TNM stage, tumor stage, lymph-node metastasis, distant metastasis, child-pugh score, vascular invasion, liver fibrosis and race using 375 HCC samples downloaded from the TCGA database. The target genes of these miRNAs were obtained using a miRNA target gene prediction database, and their functions were analyzed using the online tool DAVID.

Results: 15 miRNAs were differentially expressed in samples with venous metastasis, among which 7 were up-regulated in venous metastasis positive HCC samples. As one of the up-regulated miRNAs, hsa-miR-210 was identified as an independent prognostic factor for HCC. Using RT-qPCR, it was evident that hsa-miR-210 expression was significantly higher in venous metastasis positive HCC samples $(p=0.0036)$. Further analysis indicated that hsa-miR-210 was positively associated with AFP level, pathological grade, TNM stage, tumor stage and vascular invasion. A total of 168 hsa-miR-210 target genes, which are mainly related to tumor metastasis and tumor signaling pathways, were also predicted in this study.

Conclusion: hsa-miR-210 might promote vascular invasion of HCC cells and could be used as a prognostic biomarker.

Keywords: hsa-miR-210, venous metastasis, prognosis, hepatocellular carcinoma, bioinformatics analysis, RT-qPCR, public gene database 


\section{INTRODUCTION}

Hepatocellular carcinoma (HCC) is one of the most common gastro-intestinal cancer and one of the leading causes of cancer-related mortality worldwide (1). HCC is characterized by high malignancy, rapid infiltration, early metastasis and poor therapeutic efficacy (2). In recent years, although the diagnostic and therapeutic approaches for HCC are improved, the prognosis of HCC patients is still very poor. The HCC metastasis and recurrence remarkably reduce the survival rate and patients' quality of life. Therefore, prevention and control of HCC metastasis and recurrence are of great importance for improving the prognosis of HCC patients. With the increasingly deepened understanding of HCC pathogenesis, the microvascular invasion has become a hotspot in HCC research. Former studies suggest that microvascular invasion is a critical risk factor of HCC recurrence $(3,4)$. Therefore, identification of biomarkers to monitor vascular invasion of HCC will be substantially beneficial for HCC treatment.

MicroRNAs (miRNAs) represent small non-coding RNAs that bind to the $3^{\prime}$ untranslated regions (UTRs) of mRNA transcripts to negatively regulate target gene expression. miRNAs participate in various crucial biological processes such as cell proliferation, apoptosis, development, and differentiation $(5,6)$. miRNAs can not only be regarded as circulating biomarkers for non-invasive diagnosis and prognosis, but also therapeutic targets in diverse diseases $(7,8)$. Importantly, it has been demonstrated that miRNAs play important roles in the initiation, progression, metastasis and recurrence of many cancers (9-17).
hsa-miR-210 has been widely studied in many diseases. It has been reported that the role of hsa-miR-210 in $\mathrm{HBG} 2$ regulation and induction of $\mathrm{HbF}$ makes it valuable for the diagnosis and treatment of Sickle cell disease (18). In addition, multiple studies reveal the important function of hsa-miR-210 in hypoxia and ischemia diseases (19-21). hsa-miR-210 is also dysregulated by hypoxia in many cancers (22-25). Interestingly, hsa-miR-210 participates in the cellular immunity in the tumor microenvironment $(26,27)$. However, the research of hsamiR-210 in HCC was insufficient to date. The significance of hsa-miR-210 for the pathogenesis of HCC needs to be revealed.

In this study, we analyzed the dataset GSE6857 using the R package "limma," and found 7 significantly up-regulated miRNAs in venous metastasis positive HCC tissues. To explore whether these miRNAs are significant for the prognosis of HCC patients, the survival analysis was conducted and results showed that high hsa-miR-210 expression was correlated with a poor outcome of HCC patients. We then verified the expression of hsa-miR-210 in clinical HCC tissues using RTqPCR, result showed that hsa-miR-210 was significantly high expressed in tissues with portal vein metastasis. In the analysis with clinicopathological variables, hsa-miR-210 showed a close correlation with AFP, pathological grade, TNM stage, tumor stage and vascular invasion. Furthermore, we also identified the hsa-miR-210 target genes and their biological functions. Our data help to understand the crucial role of hsa-miR-210 in $\mathrm{HCC}$.

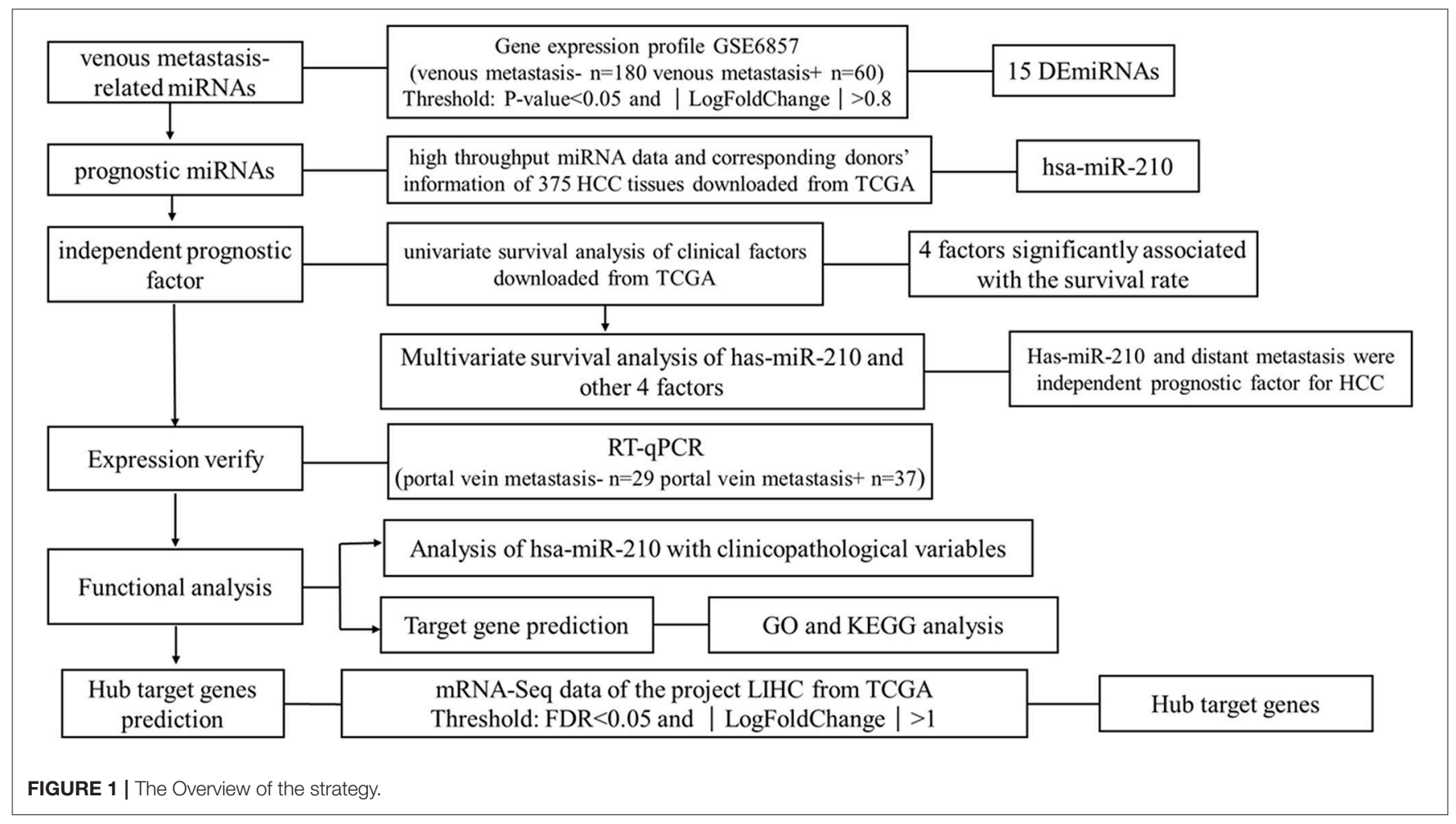




\section{MATERIALS AND METHODS}

\section{Study Design}

We downloaded one dataset from GEO database to screen the venous metastasis-related miRNAs. High throughput miRNA data and clinical information of 375 HCC tissues were downloaded from The Cancer Genome Atlas (TCGA) database to identify miRNAs which are significant for the prognosis of HCC patients. RT-qPCR was conducted to verify the expression of miRNA selected from public database. Other potential functions of selected miRNAs were also analyzed using bioinformatic analysis. The research strategy is embodied in the flowchart (Figure 1).

\section{Screening of Venous Metastasis-Related miRNAs}

Primary data and platform annotation file of dataset GSE6857 (https://www.ncbi.nlm.nih.gov/geo/query/acc.cgi? acc=GSE6857) were downloaded from GEO. The platform of this dataset is OSU-CCC MicroRNA Microarray Version 2.0 (GPL4700) (https://www.ncbi.nlm.nih.gov/geo/query/acc.cgi? acc $=$ GPL4700). The dataset contains the expression profile of 230 miRNAs in 240 paired HCC tissues and adjacent non-tumor liver tissues, 1 lymph node tissue sample of an HCC patient and 1 normal liver tissue. In this study, we chose 180 HCC tissues without venous metastasis and $60 \mathrm{HCC}$ tissues with venous metastasis to conduct the analysis. Significantly differentially expressed miRNAs were selected using R package "limma" (28), while $P<0.05$ and $\mid \log _{2}$ FoldChange $\mid>0.8$ was considered the threshold to judge differentially expressed miRNAs.

\section{Screening of Prognostic miRNAs}

The high throughput miRNA data of 375 HCC tissues and corresponding donors' information were downloaded from

TABLE 1 | Significantly differentially expressed miRNAs in venous metastasis positive HCC tissues.

\begin{tabular}{|c|c|c|c|}
\hline \multirow[t]{2}{*}{ miRNA } & \multicolumn{3}{|c|}{ Screening dataset } \\
\hline & $\log$ FC & Adj $P$-value & Type \\
\hline hsa-miR-301 & 0.842786 & 0.000267078 & Up \\
\hline hsa-let-7e & 0.847988 & $9.04 \mathrm{E}-11$ & Up \\
\hline hsa-let-7b & 0.850236 & $2.94 \mathrm{E}-12$ & Up \\
\hline hsa-miR-31 & 0.853136 & 5.34E-07 & Up \\
\hline hsa-miR-210 & 0.868472 & $1.68 \mathrm{E}-06$ & Up \\
\hline hsa-miR-371 & 0.991324 & $1.92 \mathrm{E}-10$ & Up \\
\hline hsa-miR-183 & 1.027867 & $1.14 \mathrm{E}-05$ & Up \\
\hline hsa-miR-367 & -1.08021 & $1.81 E-06$ & Down \\
\hline hsa-miR-22 & -0.9947 & 0.00055601 & Down \\
\hline hsa-miR-182 & -0.94816 & 0.00023209 & Down \\
\hline hsa-miR-100 & -0.94307 & 9.38E-07 & Down \\
\hline hsa-miR-1b-1 & -0.87813 & 0.00015521 & Down \\
\hline hsa-miR-101 & -0.84328 & 0.000209509 & Down \\
\hline hsa-miR-124a-1 & -0.81885 & 2.27E-08 & Down \\
\hline hsa-miR-101 & -0.80452 & $9.15 \mathrm{E}-10$ & Down \\
\hline
\end{tabular}

TCGA (https://cancergenome.nih.gov/). Kaplan-Meier survival analysis was conducted to find miRNAs related to the survival of HCC patients. Cox proportional hazards regression analysis was used to determine the variates which are independent prognostic factors in HCC.

\section{Verifying Using Clinical Samples}

\section{Sample Collection}

Aiming to verify the expression of miRNAs selected using public databases, we collected 37 HCC samples with portal vein metastasis and 29 HCC samples without portal vein metastasis. All the clinical specimens were obtained from patients who received liver cancer resection in Zhongnan Hospital of Wuhan University from March of 2018 to October of 2018. Each patient was diagnosed with a histopathological examination and didn't receive any treatment before surgery. Fresh samples were first incubated in RNA stable liquid (RNAlater ${ }^{\circledR}$ ) at $4^{\circ} \mathrm{C}$ for one night and then stored at $-80^{\circ} \mathrm{C}$ for further use. All the work was under approval of Institutional Review Board, and written informed consent was obtained from all participants.

\section{Real-Time Quantitative PCR (RT-qPCR) Analysis}

Total RNA was isolated using TRIzol reagent (Invitrogen, Life Technologies) and the quality of RNA was assessed by a Thermo Scientific ${ }^{\text {TM }}$ Nanodrop 2000 platform (Thermo Fisher Scientific, Waltham, MA, United States). Then reverse transcription was performed using Thermo Scientific RevertAid First Strand cDNA Synthesis Kit (Thermo Fisher Scientific, Waltham, United States). The U6 was used as the endogenous control and was amplified simultaneously with target genes. Designed PCR primers were as follows: U6: Forward, 5'-CTCGCTTCGGCAGCACA$3^{\prime}$ and Reverse, 5'-AACGCTTCACGAATTTGCGT-3'; hsa-miR210: Forward, 5'-CTGTGCGTGTGACAGC-3' and Reverse, $5^{\prime}$ GTGCAGGGTCCGAGGT- $3^{\prime}$. The reactions started at $95^{\circ} \mathrm{C}$ for $5 \mathrm{~min}$, followed by 42 cycles of $95^{\circ} \mathrm{C}$ for $30 \mathrm{~s}, 61^{\circ} \mathrm{C}$ for $30 \mathrm{~s}$, and $72^{\circ} \mathrm{C}$ for $30 \mathrm{~s}$. All experiments were carried out in duplicate for each data point. The relative expression level of hsa-miR-210 was calculated using the comparative $\mathrm{Ct}$ method formula $2^{-\Delta \mathrm{Ct}}$.

\section{Analysis of the Correlations of miRNAs With Clinicopathological Variables}

Clinical characteristics including age, gender, AFP level, pathological grade, TNM stage, tumor stage, lymph node metastasis, distant metastasis, child-pugh score, liver fibrosis, vascular invasion and race of the donors of 375 HCC tissues were downloaded from TCGA. Spearman's rank correlation was employed to analyze the relationship between miRNAs with clinicopathological variables. $P<0.05$ was considered statistically significant.

\section{miRNA Target Prediction and Functional Analysis}

miRNA target genes were predicted using 2 prediction databases including miRanda (Good mirSVR score, Conserved miRNA) (29) (www.miranda.org), and TargetScan (30) (http:// 
www.targetscan.org/vert_71/). Only genes that were confirmed by both databases were considered miRNA target genes.

To comprehensively investigate the potential roles of indicated miRNAs in HCC, GO (31) enrichment analysis and KEGG (32) analysis of miRNA target genes were conducted by the online tool DAVID (https://david.ncifcrf.gov/, version 6.8). P $<0.05$ was set as the cutoff.

\section{Hub Target Gene Prediction}

After confirming the miRNA target genes, to identify the chief mRNAs underlying the regulation mechanism of these miRNAs, high-throughput mRNA sequencing data from the LIHC project of TCGA database were used (data downloaded on March 6th, 2018). LIHC project contains 374 human HCC samples and 50 normal liver samples, in which the expression of 60244 genes was detected. Gene annotation file was downloaded from Ensembl (GRCh38) (33) database (http://www.ensembl.org/index.html) to analyze the types of these genes. Only protein-coding genes were selected for further analysis. The significantly differentially expressed mRNAs were identified using the R package "edgeR" (34) and "DESeq" (35). Benjamini-Hochberg method was used to control the false discovery rate (FDR). FDR $<0.05$ and $\mid \log _{2}$ FoldChange $\mid>1$ were set as the threshold to distinguish differentially expressed genes (DEGs). The intersection of mRNAs selected using two methods of analysis were considered as the final DEGs between HCC tissues and normal liver tissues.
Given that miRNAs negatively regulate their target genes, we took the intersection of the down-regulated genes in DEGs with the target genes of those up-regulated miRNAs, while the upregulated genes in DEGs were compared with the target genes of the down-regulated miRNAs. The mutual ones were considered hub target genes.

\section{Statistical Analysis}

Significantly differentially expressed genes in microarray data were analyzed using R package "limma," and the high throughput miRNA data of TCGA were normalized using Trimmed Mean of M-values (TMM) method by R package "edgeR." The version of R software was 3.2.5.

Kaplan-Meier survival analysis was conducted for univariate survival analysis, while Cox proportional hazards regression analysis was used for multivariate survival analysis (the analysis method is Forward:LR). The log-rank test was used to determine the difference in survival rates between two or more groups. Spearman's rank correlation was employed to analyze the relationship between miRNAs and clinicopathological variables. Differences between two group of samples were compared using Student's $t$-test or Mann Whitney test. Data were analyzed by GraphPad Prism V.6.00 software (GraphPad Software, San Diego CA, United States) and SPSS 19.0 (SPSS, Inc., Chicago, IL, United States). $P<0.05$ was considered statistically significant.
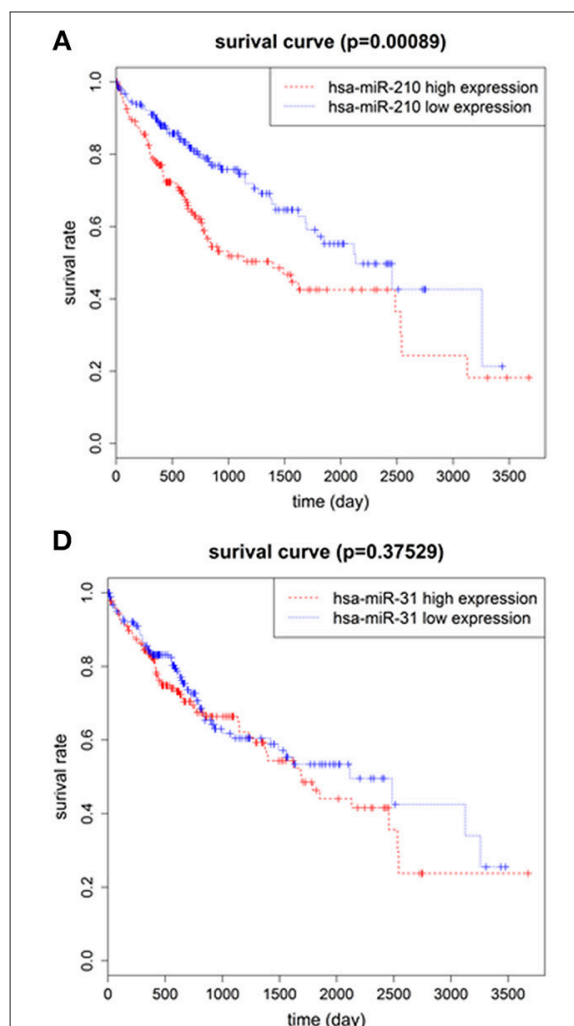

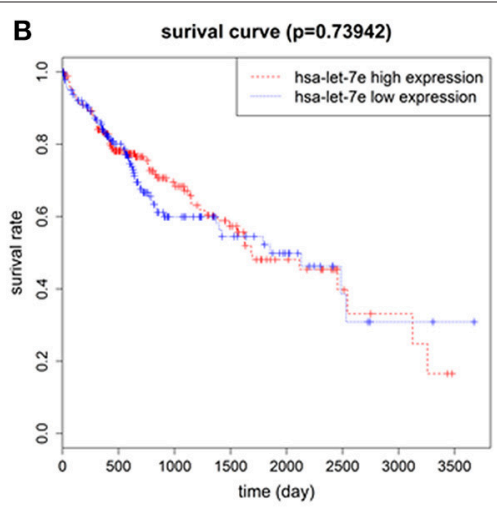

E

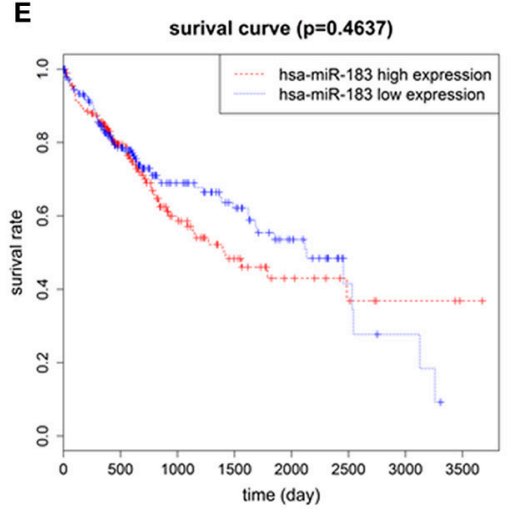

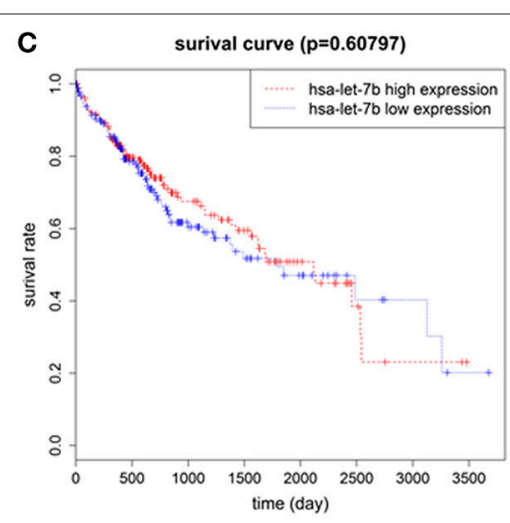

$\mathbf{F}$

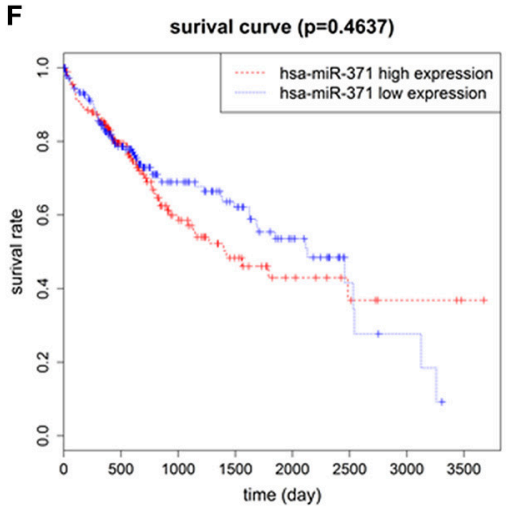

FIGURE 2 | The correlation of 6 up-regulated miRNAs with overall survival of HCC patients. Survival analysis of hsa-miR-210 (A); hsa-let-7e (B); hsa-let-7b (C); hsa-miR-31 (D); hsa-miR-183 (E); hsa-miR-371 (F). 


\section{RESULTS}

\section{Venous Metastasis-Related miRNAs in HCC}

To identify the miRNAs critical for venous metastasis in HCC, we analyzed the differentially expressed miRNAs between HCC tissues with or without venous metastasis. Based on the pre-set screening threshold, the expression of 15 miRNAs was notably different between two groups. hsa-miR-301, hsa-let-7e, hsa-let7b, hsa-miR-31, hsa-miR-210, hsa-miR-371, and hsa-miR-183 were upregulated while hsa-miR-367, hsa-miR-22, hsa-miR-182, hsa-miR-100, hsa-miR-1b-1, hsa-miR-101, hsa-miR-124a-1, and hsa-miR-101 were down-regulated in HCC tissues with venous metastasis, in comparison with those in HCC tissues without venous metastasis (Table $\mathbf{1}$ ).

\section{hsa-miR-210 Is Associated With the Prognosis of HCC Patients}

To identify the miRNAs associated with the prognosis of HCC patients, we used normalized expression of 7 up-regulated miRNAs and the survival data (overall survival time and living state) of 375 HCC patients in TCGA. We found that the overall survival rate of patients with high hsa-miR-210 expression was significantly lower than the survival rate of patients with low hsamiR-210 expression. Other miRNAs such as hsa-let-7e, hsa-let7b, hsa-miR-31, hsa-miR-371 and hsa-miR-183 had no significant influence on the survival rate of HCC patients (Figure 2). We didn't analyze hsa-miR-301 because we only found the expression of hsa-miR-301a and hsa-miR-301b in TCGA. According to miRNA nomenclature $(36,37)$, hsa-miR-301a and hsa-miR-301b are highly homologous to hsa-miR-301. They may have the same molecular function with hsa-miR-301, but there exists difference between the expression.

\section{hsa-miR-210 Is an Independent Prognostic Factor for HCC}

A multivariate survival analysis was conducted to explore whether hsa-miR-210 is an independent prognostic factor for HCC. At first, a univariate survival analysis was conducted. Clinical characteristics including age, gender, AFP level, pathological grade, TNM stage, tumor stage, lymph-node metastasis, distant metastasis, child-pugh score, liver fibrosis, vascular invasion and race were subject to Kaplan-Meier analysis to identify the factors influencing the survival rate of HCC patients. As a result, AFP, TNM stage, tumor stage and distant metastasis were significantly associated with the survival rate of HCC patients (Table 2 and Figure S1).

Cox proportional hazards regression analysis was then conducted to carry out the multivariate survival analysis. Five variates, which were demonstrated to be significant in the univariate analysis, entered the Cox hazard model to test their independent impact on HCC. Based on this analysis, high hsa-miR-210 level was identified as an independent prognostic factor for HCC (HR 2.627; 95\% CI 1.516-4.551; $p=0.001$ ). Another variate, distant metastasis, was a potential independent prognostic factor for HCC (HR 5.146; 95\% CI 1.580-16.760; $p=$ $0.007)$. However, other variates including AFP, TNM stage and tumor stage had no significant influence on the overall survival time (Table 3).

\section{hsa-miR-210 Up-Regulate in Portal Vein Metastasis HCC Samples}

Based on the previous analysis using public database, we found high hsa-miR-210 expression in venous metastasis HCC tissues. Aiming to verify this expression trend, we conducted RT-qPCR assay on 37 HCC samples with portal vein metastasis and 29 HCC samples without venous metastasis. The results showed that

TABLE 2 | Univariate analysis of factors affecting overall survival of HCC patients.

\begin{tabular}{|c|c|c|c|}
\hline Variable & Group & Case number & $P$ \\
\hline \multirow[t]{2}{*}{ Age } & $<50$ & 71 & 0.173 \\
\hline & $\geq 50$ & 301 & \\
\hline \multirow[t]{2}{*}{ Gender } & Male & 253 & 0.309 \\
\hline & Female & 119 & \\
\hline \multirow[t]{2}{*}{ AFP } & $<20$ & 150 & 0.019 \\
\hline & $\geq 20$ & 130 & \\
\hline \multirow[t]{4}{*}{ Grade } & 1 & 55 & 0.858 \\
\hline & 2 & 176 & \\
\hline & 3 & 123 & \\
\hline & 4 & 13 & \\
\hline \multirow[t]{4}{*}{ TNM stage } & I & 173 & 0.000 \\
\hline & ॥ & 85 & \\
\hline & III & 85 & \\
\hline & IV & 5 & \\
\hline \multirow[t]{4}{*}{ Tumor stage } & $\mathrm{T} 1$ & 183 & 0.000 \\
\hline & T2 & 93 & \\
\hline & T3 & 80 & \\
\hline & T4 & 13 & \\
\hline \multirow[t]{2}{*}{ Lymph-node metastasis } & NO & 254 & 0.317 \\
\hline & N1 & 4 & \\
\hline \multirow[t]{2}{*}{ Distant metastasis } & MO & 269 & 0.01 \\
\hline & M1 & 4 & \\
\hline \multirow[t]{3}{*}{ Child-pugh } & A & 219 & 0.357 \\
\hline & B & 21 & \\
\hline & C & 1 & \\
\hline \multirow[t]{2}{*}{ Liver fibrosis } & No & 75 & 0.316 \\
\hline & Yes & 139 & \\
\hline \multirow[t]{2}{*}{ Vascular invasion } & No & 206 & 0.162 \\
\hline & Yes & 110 & \\
\hline \multirow[t]{2}{*}{ Race } & Asian & 160 & 0.129 \\
\hline & Others & 202 & \\
\hline
\end{tabular}

Note: The statistically significant values are in bold.

TABLE 3 | Cox proportional hazards regression analysis of factors affecting overall survival of HCC patients.

\begin{tabular}{lccccc}
\hline Variable & B & SE & Sig. & HR & 95\% Cl \\
\hline hsa-miR-210 & 0.966 & 0.280 & 0.001 & 2.627 & $1.516-4.551$ \\
Distant metastasis & 1.638 & 0.602 & 0.007 & 5.146 & $1.580-16.760$
\end{tabular}


the mean value of hsa-miR-210 in portal vein metastasis positive tissues and negative HCC tissues were 0.00098 vs. 0.00032 , respectively $(P=0.0036)$. The finding suggests that expression of hsa-miR-210 was significantly higher in HCC samples with portal vein metastasis (Figure 3).

\section{The Correlation of hsa-miR-210 With HCC Clinicopathological Variables}

To clarify the relationship between HCC and hsa-miR210, we analyzed the correlation of hsa-miR-210 with HCC clinicopathological variables.

Notably, our analysis showed that hsa-miR-210 expression was positively associated with vascular invasion. What's more, high expression of has-miR-210 portends high AFP level, pathological grade, TNM stage and tumor stage, which verified the essential role of hsa-miR-210 in influencing the degree of tumor malignant. But its expression had no significant correlation with age, gender, Lymph-node metastasis, distant metastasis, child-pugh, liver fibrosis and race (Table 4 and Figure S2).

\section{Target Gene Prediction and Functional Analysis}

To provide deep insight into the role of hsa-miR-210 in the HCC initiation and progression, hsa-miR-210 target genes were predicted by two prediction databases. 684 target genes and 4,059 target genes were found from miRanda database and TargetScan database, respectively. Among them, a total of 168 common genes were identified as hsa-miR-210 target genes (Table S1). In particular, 15 predicted target genes have been indicated to be regulated by hsa-miR-210 in former studies.

Regarding the function and pathway enrichment analysis of the target genes, the GO analysis of the hsa-miR-210 target

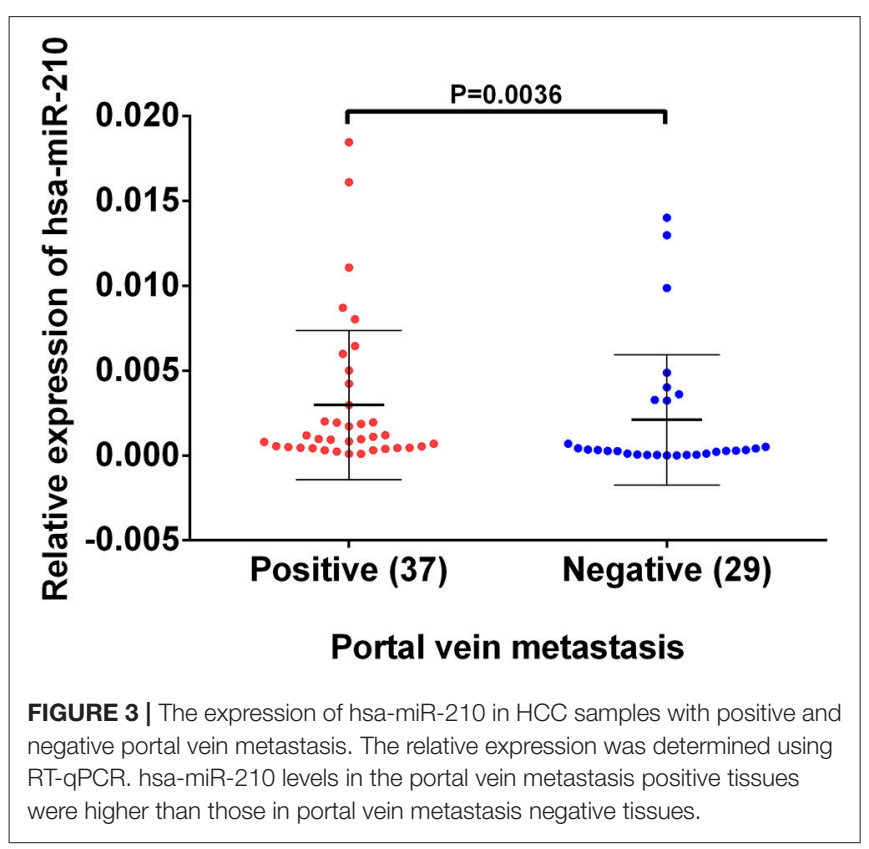

genes was summarized in Figure 4. The significantly enriched items in each domain were demonstrated according to their p-values. hsa-miR-210 target genes were mainly enriched in the biological processes including blood vessel development, protein autophosphorylation, platelet activation and negative regulation of transcription from RNA polymerase II promoter. The products of these target genes are involved in various functions including receptor signaling protein serine/threonine kinase activity, fibroblast growth factor-activated receptor activity and protein complex binding. Moreover, they are localized in the nucleus or cytoplasm. KEGG analysis revealed that hsa-miR-210 target genes mainly participate in cancerrelated signaling pathways like Ras signaling, TGF- $\beta$ signaling, phosphatidylinositol signaling, and central carbon metabolism. They were also involved in the signaling pathways regulating stem cell pluripotency (Figure 5). Notably, INHBB, ACVR1B, MAPK1, FGFR1, and PIK3R5 were involved in multiple pathways.

\section{Hub Target Genes of hsa-miR-210}

To identify the chief mRNAs that participate in the regulation of hsa-miR-210, the high-throughput mRNA sequencing data from TCGA was introduced. The differential expression analysis using R package "edgeR" revealed 4895 differentially

TABLE 4 | The relationship between hsa-mir-210 and the clinicopathological variables of $\mathrm{HCC}$.

\begin{tabular}{|c|c|c|c|c|}
\hline & \multirow[t]{2}{*}{ Group } & \multirow[t]{2}{*}{ Case } & \multicolumn{2}{|c|}{ hsa-miR-210 } \\
\hline & & & $R$ & $p$ \\
\hline \multirow[t]{2}{*}{ Age } & $<50$ & 71 & 0.072 & 0.164 \\
\hline & $\geq 50$ & 300 & & \\
\hline \multirow[t]{2}{*}{ Gender } & Male & 253 & 0.093 & 0.074 \\
\hline & Female & 119 & & \\
\hline \multirow[t]{2}{*}{ AFP } & $<20$ & 150 & 0.095 & 0.112 \\
\hline & $\geq 20$ & 131 & & \\
\hline \multirow[t]{2}{*}{ Grade } & 1 & 55 & 0.144 & 0.006 \\
\hline & $2-4$ & 313 & & \\
\hline \multirow[t]{2}{*}{ TNM stage } & $1-I 1$ & 258 & 0.197 & 0.00 \\
\hline & III-IV & 90 & & \\
\hline \multirow[t]{2}{*}{ Tumor stage } & T0-T1 & 182 & 0.162 & 0.002 \\
\hline & T2-T4 & 187 & & \\
\hline \multirow[t]{2}{*}{ Lymph-node metastasis } & NO & 254 & 0.061 & 0.332 \\
\hline & N1 & 4 & & \\
\hline \multirow[t]{2}{*}{ Distant metastasis } & MO & 269 & -0.005 & 0.934 \\
\hline & M1 & 4 & & \\
\hline \multirow[t]{2}{*}{ Child-pugh } & A & 220 & 0.028 & 0.67 \\
\hline & $B-C$ & 22 & & \\
\hline \multirow[t]{2}{*}{ Liver fibrosis } & No & 75 & -0.087 & 0.206 \\
\hline & Yes & 140 & & \\
\hline \multirow[t]{2}{*}{ Vascular invasion } & No & 206 & 0.135 & 0.016 \\
\hline & Yes & 111 & & \\
\hline \multirow[t]{2}{*}{ Race } & Asian & 160 & -0.024 & 0.647 \\
\hline & Others & 202 & & \\
\hline
\end{tabular}

Note: The statistically significant values are in bold. 

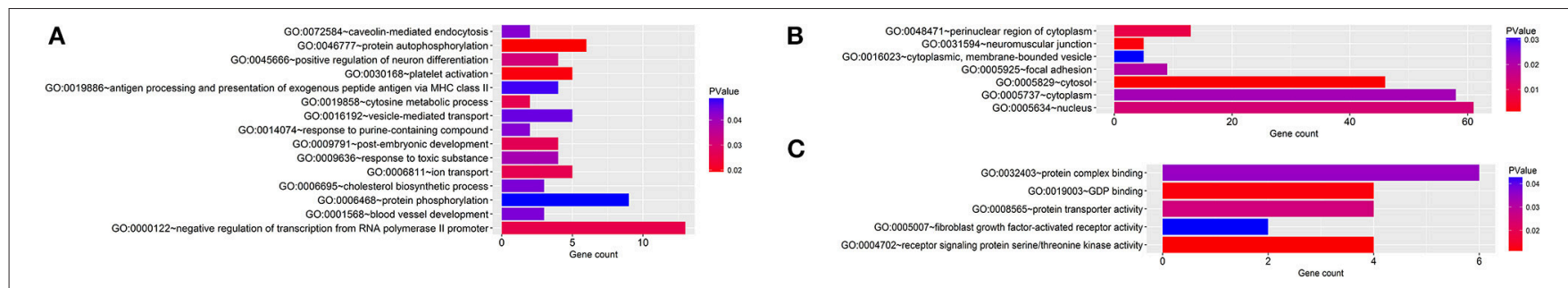

FIGURE 4 | GO analysis of hsa-miR-210 target genes. GO enrichment of target genes in biological process ontology (A), cellular component ontology (B) and molecular function ontology (C).

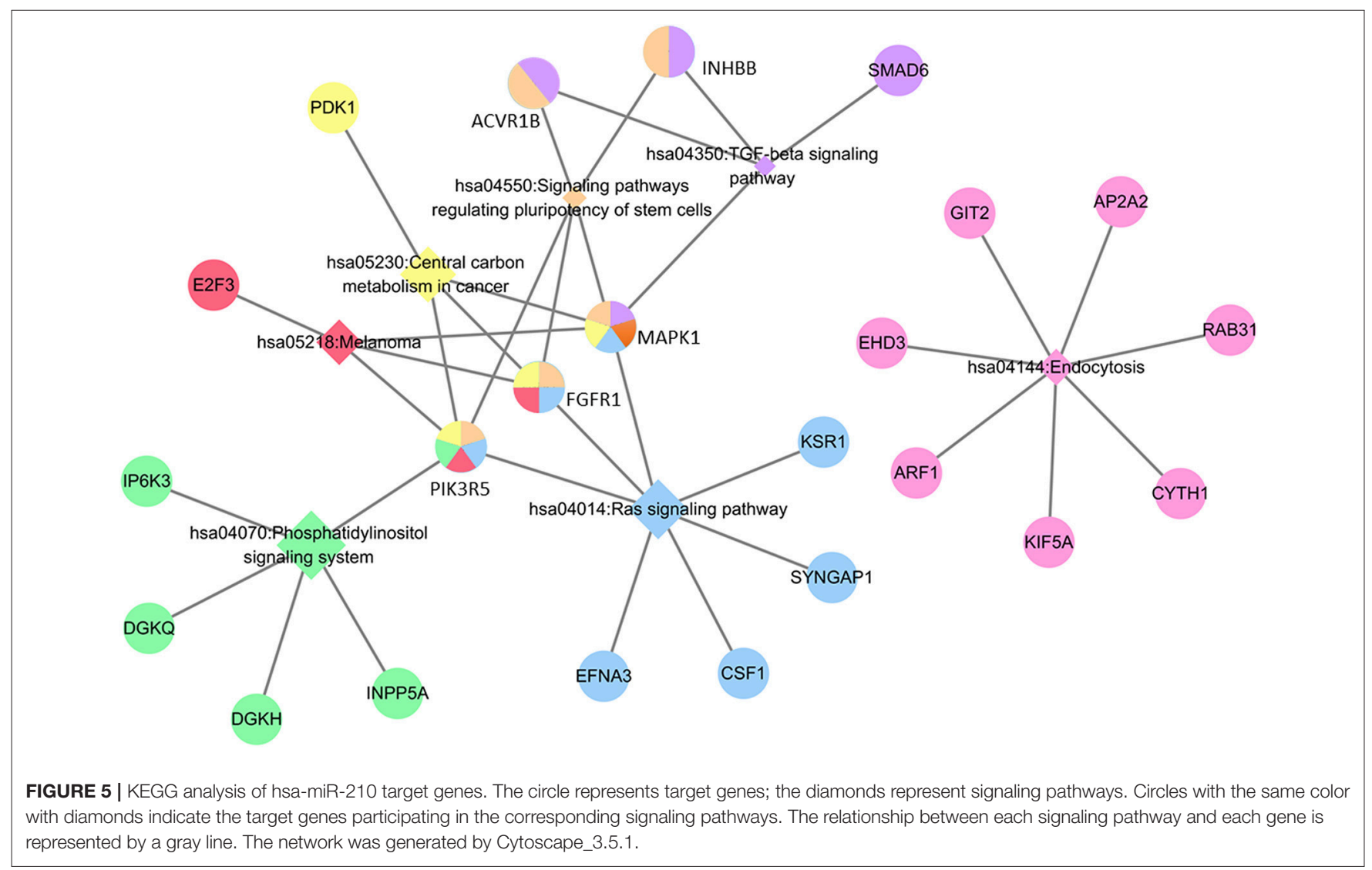

expressed genes. Among them, 3847 genes were up-regulated while 1048 genes were down-regulated (Figure 6A). 2173 mRNAs were obtained using differential expression analysis conducted by R package "DESeq", thereinto, 1290 genes were up-regulated while 883 genes were down-regulated (Figure 6B). After taking the intersection of the result of two analysis, we got 1290 mRNAs up regulated in HCC tissues (Figure 6C) and 793 mRNAs significantly down regulated (Figure 6D).

The intersection between the differentially expressed genes and the predicted hsa-miR-210 target genes was set according to the negative correlation between miRNAs and their target genes. hsa-miR-210, which was up-regulated in HCC, significantly down-regulated 7 target genes (JDP2, SAA1, CR1, SHMT1, $\mathrm{KMO}$, NOL4, and EHD3) in HCC tissues.

\section{DISCUSSION}

Metastasis is one of the main malignant characters of cancers. It is responsible for up to $90 \%$ of cancer-related mortality (38). Metastasis involves a series of complex cascades including cancer cell invasion into the surrounding tissue, infiltration across blood vessels and lymphatic vessels, migration to distant tissues and organs, colonization in distant tissues and organs (39). The vascular invasion has been proven to be an important indicator of HCC recurrence after hepatectomy and liver resection (40). Early detection and treatment of vascular invasion could help reduce the recurrence and prolong the survival of patients.

As a hypoxia-inducible factor, hsa-miR-210 has been confirmed by multiple studies to play a crucial role in tumor proliferation, metastasis and invasion. High hsa-miR-210 


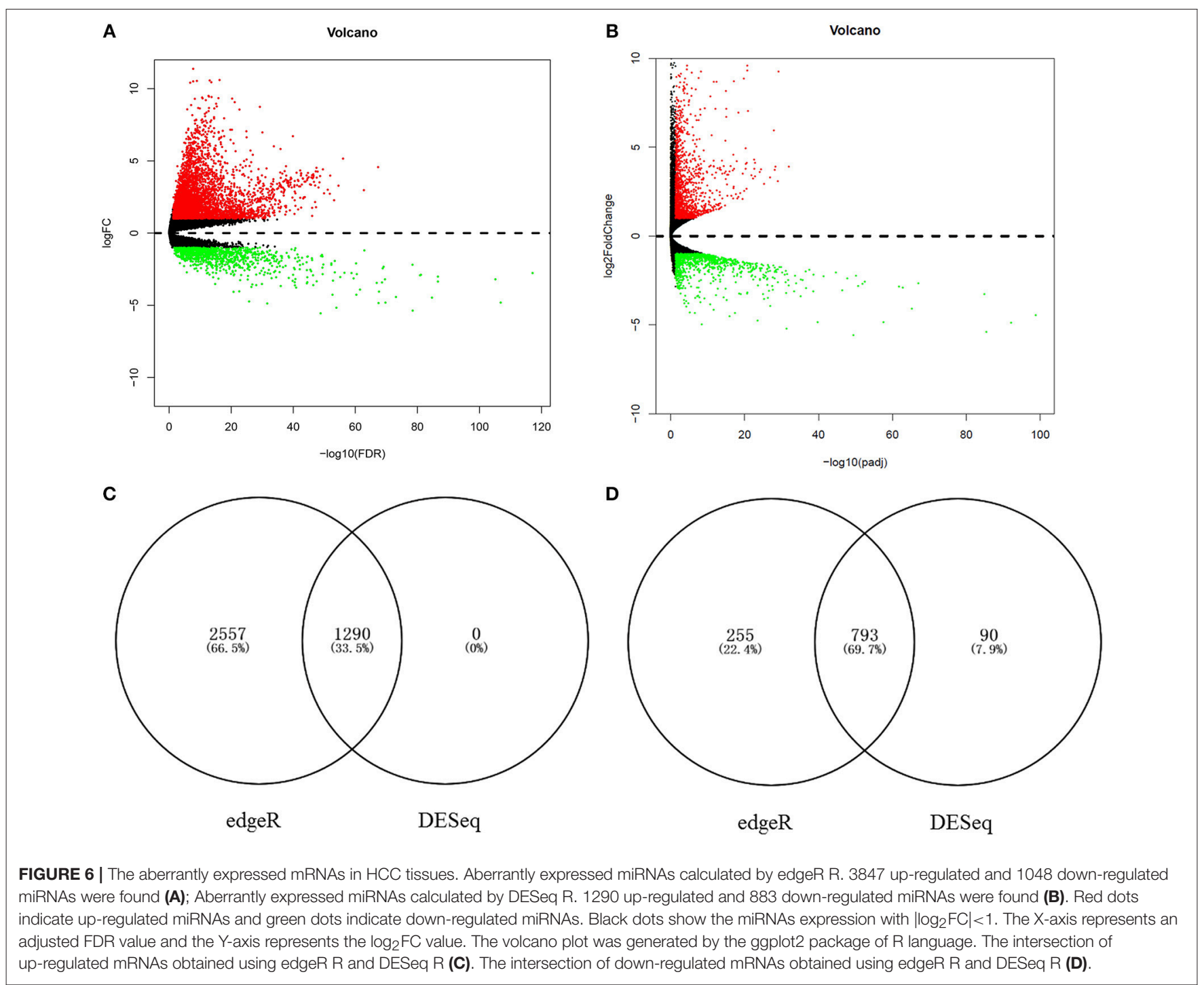

expression promotes the metastasis and indicates poor prognosis of gastric cancer and colorectal cancer $(41,42)$. hsa-miR-210 also plays a central role in tumor growth through participating in the induction of cell senescence, regulation of the recruitment of inflammatory cells, formation of tumor blood vessels, and generation of high-energy metabolites to maintain (43). Moreover, hsa-mir-210 is also used to assess drug susceptibility or as a therapeutic target (44).

In this study, through analyzing the miRNA expression profile of HCC tissues with or without venous metastasis, we found that hsa-miR-210 was significantly increased in venous metastasis positive samples. Moreover, high hsa-miR-210 expression was closely correlated with poor HCC outcome in both univariate and multivariate survival analysis. Additionally, hsa-miR-210 expression was positively correlated with AFP, pathological grade, TNM stage, tumor stage and vascular invasion, suggesting that hsa-miR-210 indicate the extent of HCC malignancy. It has been proposed that hsa-miR-210 promotes the metastasis of hypoxic
HCC cells by directly targeting the metastasis suppressor vacuole membrane protein 1 (VMP1) (45). Meanwhile, it was reported that high hsa-miR-210 expression is closely associated with the poor prognosis including short tumor-free survival time and low survival rate (46). Previous studies also demonstrated that hsamiR-210 promotes HCC angiogenesis through downregulating FGFRL1. Additionally, an in vitro research confirmed that high hsa-mir-210 expression promotes tumor cell proliferation, inhibits apoptosis and reduces the radiosensitivity of HCC cells in low oxygen environment (47). To our knowledge, we are the first to unveil that hsa-miR-210 promotes vascular invasion in HCC through bioinformatics analysis.

To explore the biological function of hsa-miR-210 and the signaling pathways it regulates, we used two miRNA target gene prediction databases to obtain hsa-miR-210 target genes. Among these genes, the expression of BDNF, ZNF462, GIT2, GPD1L, ISCU, NFIC, FGFRL1, MNT, EFNA3, and E2F3 have been shown to be regulated by hsa-miR-210 in previous studies 
(48-56). Other target genes which haven't been validated by experiments might be clues for exploring new biological functions of hsa-miR-210 in HCC. In GO and KEGG analysis, we found that the biological processes and molecular functions of these target genes were closely related to malignancy and metastasis of tumors. Additionally, hsa-miR-210 was involved in many tumorigenesis-related signaling pathways, such as Ras signaling (57), TGF- $\beta$ signaling (58), and phosphatidylinositol signaling $(59,60)$. Particularly, the regulatory effects of hsamiR-210 on TGF- $\beta$ signaling and phosphatidylinositol signaling have been confirmed previously $(61,62)$. hsa-miR-210 also influences the signaling pathways involved in stem cell pluripotency and central carbon metabolism. In our research, we found that MAPK1, FGFR1, and PIK3R5 participate in more than 4 important cancer-related signaling pathways. Nonetheless, no study has shown the effect of hsa-miR-210 on their expression. Therefore, investigation on these genes will facilitate a deeper understanding of hsa-miR-210 in HCC. In addition, the molecular mechanisms by which hsa-mir-210 promotes HCC vascular invasion requires further investigation, and so is the case for other candidate target genes of hsa-miR-210.

In conclusion, this study demonstrates that hsa-mir-210 promote venous metastasis in HCC. hsa-mir-210 may be a

\section{REFERENCES}

1. Kassebaum NJ, Bertozzi-Villa A, Coggeshall MS, Shackelford KA, Steiner $\mathrm{C}$, Heuton $\mathrm{KR}$, et al. Global, regional, and national levels and causes of maternal mortality during 1990-2013: a systematic analysis for the Global Burden of Disease Study 2013. Lancet (2014) 384:980-1004. doi: 10.1016/s0140-6736(14)60696-6

2. Bruix J, Sherman M. Management of hepatocellular carcinoma. Hepatology (2005) 42:1208-36. doi: 10.1002/hep.20933

3. Goh BK, Chow PK, Teo JY, Wong JS, Chan CY, Cheow PC, et al. Number of nodules, Child-Pugh status, margin positivity, and microvascular invasion, but not tumor size, are prognostic factors of survival after liver resection for multifocal hepatocellular carcinoma. J Gastrointest Surg. (2014) 18:1477-85. doi: 10.1007/s11605-014-2542-0

4. Sumie S, Kuromatsu R, Okuda K, Ando E, Takata A, Fukushima N, et al. Microvascular invasion in patients with hepatocellular carcinoma and its predictable clinicopathological factors. Ann Surg Oncol. (2008) 15:1375-82. doi: 10.1245/s10434-008-9846-9

5. Bartel DP. MicroRNAs: genomics, biogenesis, mechanism, and function. Cell (2004) 116:281-97. doi: 10.1016/S0092-8674(04)00045-5

6. Ambros V. The functions of animal microRNAs. Nature (2004) 431:350-5. doi: $10.1038 /$ nature 02871

7. Wong CM, Wong CC, Lee JM, Fan DN, Au SL, Ng IO. Sequential alterations of microRNA expression in hepatocellular carcinoma development and venous metastasis. Hepatology (2012) 55:1453-61. doi: 10.1002/hep.25512

8. Xia H, Ooi LL, Hui KM. MicroRNA-216a/217-induced epithelialmesenchymal transition targets PTEN and SMAD7 to promote drug resistance and recurrence of liver cancer. Hepatology (2013) 58:629-41. doi: 10.1002/hep.26369

9. Lewis BP, Shih IH, Jones-Rhoades MW, Bartel DP, Burge CB. Prediction of mammalian microRNA targets. Cell (2003) 115:787-98. doi: 10.1016/S0092-8674(03)01018-3

10. Lin S, Gregory RI. MicroRNA biogenesis pathways in cancer. Nat Rev Cancer (2015) 15:321-33. doi: 10.1038/nrc3932

11. Calin GA, Liu CG, Sevignani C, Ferracin M, Felli N, Dumitru CD, et al. MicroRNA profiling reveals distinct signatures in $\mathrm{B}$ cell chronic promising biomarker for evaluating the prognosis of $\mathrm{HCC}$ patients.

\section{AUTHOR CONTRIBUTIONS}

All authors listed have made a substantial, direct and intellectual contribution to the work, and approved it for publication.

\section{ACKNOWLEDGMENTS}

This work was supported by Science and Technology Innovation Fostering Foundation of Zhongnan Hospital of Wuhan University (cxpy20160025, cxpy2017080), and National Natural Science Foundation of China (No. 81672114). This work was also funded by Applied Basic Research Program of Science and Technology Bureau Foundation of Wuhan (No. 2016060101010054) and Wuhan City health and family planning medical talented youth development project.

\section{SUPPLEMENTARY MATERIAL}

The Supplementary Material for this article can be found online at: https://www.frontiersin.org/articles/10.3389/fonc. 2018.00569/full\#supplementary-material lymphocytic leukemias. Proc Natl Acad Sci USA. (2004) 101:11755-60. doi: $10.1073 /$ pnas.0404432101

12. Carleton M, Cleary MA, Linsley PS. MicroRNAs and cell cycle regulation. Cell Cycle (2007) 6:2127-32. doi: 10.4161/cc.6.17.4641

13. Calin GA, Sevignani C, Dumitru CD, Hyslop T, Noch E, Yendamuri S, et al. Human microRNA genes are frequently located at fragile sites and genomic regions involved in cancers. Proc Nat Acad Sci. (2004) 101:29993004. doi: 10.1073/pnas.0307323101

14. Farazi TA, Spitzer JI, Morozov P, Tuschl T. miRNAs in human cancer. J Pathol. (2011) 223:102-15. doi: 10.1002/path.2806

15. Aleckovic M, Kang Y. Regulation of cancer metastasis by cell-free miRNAs. Biochim Biophys Acta (2015) 1855:24-42. doi: 10.1016/j.bbcan.2014. 10.005

16. Ding XM. MicroRNAs: regulators of cancer metastasis and epithelialmesenchymal transition (EMT). Chin J Cancer (2014) 33:140-7. doi: $10.5732 /$ cjc. 013.10094

17. Bouyssou JM, Manier S, Huynh D, Issa S, Roccaro AM, Ghobrial IM. Regulation of microRNAs in cancer metastasis. Biochim Biophys Acta (2014) 1845:255-65. doi: 10.1016/j.bbcan.2014.02.002

18. Sawant M, S C, Colah R, Ghosh K, Nadkarni A. Does HbF induction by hydroxycarbamide work through MIR210 in sickle cell anaemia patients? $\mathrm{Br} \mathrm{J}$ Haematol. (2016) 173:801-3. doi: 10.1111/bjh.13642

19. Ma Q, Dasgupta C, Li Y, Bajwa NM, Xiong F, Harding B, et al. Inhibition of microRNA-210 provides neuroprotection in hypoxic-ischemic brain injury in neonatal rats. Neurobiol Dis. (2016) 89:202-12. doi: 10.1016/j.nbd.2016. 02.011

20. White K, Lu Y, Annis S, Hale AE, Chau BN, Dahlman JE, et al. Genetic and hypoxic alterations of the microRNA-210-ISCU1/2 axis promote iron-sulfur deficiency and pulmonary hypertension. EMBO Mol Med. (2015) 7:695-713. doi: 10.15252/emmm.201404511

21. Jiang Y, Li L, Tan X, Liu B, Zhang Y, Li C. miR-210 mediates vagus nerve stimulation-induced antioxidant stress and anti-apoptosis reactions following cerebral ischemia/reperfusion injury in rats. J Neurochem. (2015) 134:173-81. doi: $10.1111 /$ jnc. 13097

22. Kiga K, Mimuro H, Suzuki M, Shinozaki-Ushiku A, Kobayashi T, Sanada T, et al. Epigenetic silencing of miR-210 increases the proliferation of gastric 
epithelium during chronic Helicobacter pylori infection. Nat Commun. (2014) 5:4497. doi: 10.1038/ncomms5497

23. Camps C, Buffa FM, Colella S, Moore J, Sotiriou C, Sheldon H, et al. hsa-miR-210 Is induced by hypoxia and is an independent prognostic factor in breast cancer. Clin Cancer Res. (2008) 14:1340-8. doi: 10.1158/1078-0432.ccr-07-1755

24. Cui H, Seubert B, Stahl E, Dietz H, Reuning U, Moreno-Leon L, et al. Tissue inhibitor of metalloproteinases-1 induces a pro-tumourigenic increase of miR-210 in lung adenocarcinoma cells and their exosomes. Oncogene (2015) 34:3640-50. doi: 10.1038/onc.2014.300

25. Noman MZ, Janji B, Hu S, Wu JC, Martelli F, Bronte V, et al. Tumorpromoting effects of myeloid-derived suppressor cells are potentiated by hypoxia-induced expression of miR-210. Cancer Res. (2015) 75:3771-87. doi: 10.1158/0008-5472.can-15-0405

26. Noman MZ, Buart S, Romero P, Ketari S, Janji B, Mari B, et al. Hypoxia-inducible miR-210 regulates the susceptibility of tumor cells to lysis by cytotoxic $T$ cells. Cancer Res. (2012) 72:4629-41. doi: 10.1158/0008-5472.can-12-1383

27. Markou A, Zavridou M, Sourvinou I, Yousef G, Kounelis S, Malamos $\mathrm{N}$, et al. Direct comparison of metastasis-related miRNAs expression levels in circulating tumor cells, corresponding plasma, and primary tumors of breast cancer patients. Clin Chem. (2016) 62:1002-11. doi: 10.1373/clinchem.2015.253716

28. Gentleman R, Carey VJ, Huber W. Bioinformatics and Computational Biology Solutions Using $R$ and Bioconductor. New York, NY: Springer Science+Business Media (2005). p. 388-9.

29. Betel D, Koppal A, Agius P, Sander C, Leslie C. Comprehensive modeling of microRNA targets predicts functional non-conserved and non-canonical sites. Genome Biol. (2010) 11:R90. doi: 10.1186/gb-2010-11-8-r90

30. Lewis BP, Burge CB, Bartel DP. Conserved seed pairing, often flanked by adenosines, indicates that thousands of human genes are microRNA targets. Cell (2005) 120:15-20. doi: 10.1016/j.cell.2004.12.035

31. Ashburner M, Ball CA, Blake JA, Botstein D, Butler H, Cherry JM, et al. Gene ontology: tool for the unification of biology. The Gene Ontology Consortium. Nat Genet. (2000) 25:25-9. doi: 10.1038/75556

32. Ogata H, Goto S, Sato K, Fujibuchi W, Bono H, Kanehisa M. KEGG: kyoto encyclopedia of genes and genomes. Nucleic Acids Res. (1999) 27:29-34. doi: 10.1093/nar/27.1.29

33. Cunningham F, Amode MR, Barrell D, Beal K, Billis K, Brent S, et al. Ensembl 2015. Nucleic Acids Res. (2015) 43:D662-9. doi: 10.1093/nar/gku1010

34. Robinson MD, McCarthy DJ, Smyth GK. edgeR: a Bioconductor package for differential expression analysis of digital gene expression data. Bioinformatics (2010) 26:139-40. doi: 10.1093/bioinformatics/btp616

35. Anders S, Huber W. Differential expression analysis for sequence count data. Genome Biol. (2010) 11:R106. doi: 10.1186/gb-2010-11-10-r106

36. Lagos-Quintana M, Rauhut R, Lendeckel W, Tuschl T. Identification of novel genes coding for small expressed RNAs. Science (2001) 294:853-8. doi: $10.1126 /$ science. 1064921

37. Griffiths-Jones S, Hui JH, Marco A, Ronshaugen M. MicroRNA evolution by arm switching. EMBO Rep. (2011) 12:172-7. doi: 10.1038/embor.2010.191

38. Chaffer CL, Weinberg RA. A perspective on cancer cell metastasis. Science (2011) 331:1559-64. doi: 10.1126/science.1203543

39. Fidler IJ. The pathogenesis of cancer metastasis: the 'seed and soil' hypothesis revisited. Nat Rev Cancer (2003) 3:453-8. doi: 10.1038/nrc1098

40. Iguchi T, Shirabe K, Aishima S, Wang H, Fujita N, Ninomiya M, et al. New pathologic stratification of microvascular invasion in hepatocellular carcinoma: predicting prognosis after living-donor liver transplantation. Transplantation (2015) 99:1236-42. doi: 10.1097/tp.00000000000 00489

41. Zhang C, Tian W, Meng L, Qu L, Shou C. PRL-3 promotes gastric cancer migration and invasion through a NF-kappaB-HIF-1alpha-miR-210 axis. $J$ Mol Med. (2016) 94:401-15. doi: 10.1007/s00109-015-1350-7

42. Qu A, Du L, Yang Y, Liu H, Li J, Wang L, et al. Hypoxia-inducible MiR210 is an independent prognostic factor and contributes to metastasis in colorectal cancer. PLoS ONE (2014) 9:e90952. doi: 10.1371/journal.pone.00 90952
43. Taddei ML, Cavallini L, Comito G, Giannoni E, Folini M, Marini A, et al. Senescent stroma promotes prostate cancer progression: the role of miR-210. Mol Oncol. (2014) 8:1729-46. doi: 10.1016/j.molonc.2014.07.009

44. Mei Y, Gao C, Wang K, Cui L, Li W, Zhao X, et al. Effect of microRNA-210 on prognosis and response to chemotherapeutic drugs in pediatric acute lymphoblastic leukemia. Cancer Sci. (2014) 105:463-72. doi: $10.1111 /$ cas. 12370

45. Ying Q, Liang L, Guo W, Zha R, Tian Q, Huang S, et al. Hypoxia-inducible microRNA-210 augments the metastatic potential of tumor cells by targeting vacuole membrane protein 1 in hepatocellular carcinoma. Hepatology (2011) 54:2064-75. doi: 10.1002/hep.24614

46. Yang $\mathrm{Y}$, Zhang J, Xia T, Li G, Tian T, Wang M, et al. MicroRNA210 promotes cancer angiogenesis by targeting fibroblast growth factor receptor-like 1 in hepatocellular carcinoma. Oncol Rep. (2016) 36:2553-62. doi: 10.3892/or.2016.5129

47. Yang W, Sun T, Cao J, Liu F, Tian Y, Zhu W. Downregulation of miR-210 expression inhibits proliferation, induces apoptosis and enhances radiosensitivity in hypoxic human hepatoma cells in vitro. Exp Cell Res. (2012) 318:944-54. doi: 10.1016/j.yexcr.2012. 02.010

48. Fasanaro P, Greco S, Lorenzi M, Pescatori M, Brioschi M, Kulshreshtha $\mathrm{R}$, et al. An integrated approach for experimental target identification of hypoxia-induced miR-210. J Biol Chem. (2009) 284:35134-43. doi: $10.1074 /$ jbc.M109.052779

49. Chen WY, Liu WJ, Zhao YP, Zhou L, Zhang TP, Chen G, et al. Induction, modulation and potential targets of miR-210 in pancreatic cancer cells. Hepatobil Pancreat Dis Int. (2012) 11:319-24. doi: 10.1016/S1499-3872(12)60168-4

50. Kelly TJ, Souza AL, Clish CB, Puigserver P. A hypoxia-induced positive feedback loop promotes hypoxia-inducible factor lalpha stability through miR-210 suppression of glycerol-3-phosphate dehydrogenase 1-like. Mol Cell Biol. (2011) 31:2696-706. doi: 10.1128/mcb.01 242-10

51. Lee DC, Romero R, Kim JS, Tarca AL, Montenegro D, Pineles BL, et al. miR-210 targets iron-sulfur cluster scaffold homologue in human trophoblast cell lines: siderosis of interstitial trophoblasts as a novel pathology of preterm preeclampsia and small-for-gestational-age pregnancies. Am J Pathol. (2011) 179:590-602. doi: 10.1016/j.ajpath.2011. 04.035

52. Zhang H, Mai Q, Chen J. MicroRNA-210 is increased and it is required for dedifferentiation of osteosarcoma cell line. Cell Biol Int. (2017) 41:267-75. doi: $10.1002 /$ cbin. 10721

53. Tsuchiya S, Fujiwara T, Sato F, Shimada Y, Tanaka E, Sakai Y, et al. MicroRNA-210 regulates cancer cell proliferation through targeting fibroblast growth factor receptor-like 1 (FGFRL1). J Biol Chem. (2011) 286:420-8. doi: 10.1074/jbc.M110.170852

54. Zhang Z, Sun H, Dai H, Walsh RM, Imakura M, Schelter J, et al. MicroRNA miR-210 modulates cellular response to hypoxia through the MYC antagonist MNT. Cell Cycle (2009) 8:2756-68. doi: 10.4161/cc.8.17.9387

55. Wang Z, Yin B, Wang B, Ma Z, Liu W, Lv G. MicroRNA-210 promotes proliferation and invasion of peripheral nerve sheath tumor cells targeting EFNA3. Oncol Res. (2013) 21:145-54. doi: 10.3727/096504013x138413406 89573

56. Gou D, Ramchandran R, Peng X, Yao L, Kang K, Sarkar J, et al. miR210 has an antiapoptotic effect in pulmonary artery smooth muscle cells during hypoxia. Am J Physiol Lung Cell Mol Physiol. (2012) 303:L682-91. doi: 10.1152/ajplung.00344.2011

57. Malaney S, Daly RJ. The ras signaling pathway in mammary tumorigenesis and metastasis. J Mammary Gland Biol Neoplasia (2001) 6:101-13. doi: 10.1023/A:1009572700317

58. Wakefield LM, Hill CS. Beyond TGFbeta: roles of other TGFbeta superfamily members in cancer. Nat Rev Cancer (2013) 13:328-41. doi: 10.1038/ $\operatorname{nrc3500}$

59. Riehle RD, Cornea S, Degterev A. Role of phosphatidylinositol 3,4,5trisphosphate in cell signaling. Adv Exp Med Biol. (2013) 991:105-39. doi: 10.1007/978-94-007-6331-9_7 
60. Woolley JF, Dzneladze I, Salmena L. Phosphoinositide signaling in cancer: INPP4B Akt(s) out. Trends Mol Med. (2015) 21:530-2. doi: 10.1016/j.molmed.2015.06.006

61. Lee DW, Futami M, Carroll M, Feng Y, Wang Z, Fernandez M, et al. Loss of SHIP-1 protein expression in high-risk myelodysplastic syndromes is associated with miR-210 and miR-155. Oncogene (2012) 31:4085-94. doi: 10.1038/onc.2011.579

62. Dai Y, Ren D, Yang Q, Cui Y, Guo W, Lai Y, et al. The TGFbeta signalling negative regulator PICK1 represses prostate cancer metastasis to bone. Br J Cancer (2017) 117:685-94. doi: 10.1038/bjc. 2017.212
Conflict of Interest Statement: The authors declare that the research was conducted in the absence of any commercial or financial relationships that could be construed as a potential conflict of interest.

Copyright (c) $2018 \mathrm{Ji}$, Rong, Luo, Li, Jiang, Weng, Chen, Zhang, Xie and Wang. This is an open-access article distributed under the terms of the Creative Commons Attribution License (CC BY). The use, distribution or reproduction in other forums is permitted, provided the original author(s) and the copyright owner(s) are credited and that the original publication in this journal is cited, in accordance with accepted academic practice. No use, distribution or reproduction is permitted which does not comply with these terms. 